\title{
Modeling of near-continuum laminar boundary layer shocks using DSMC
}

Ozgur Tumuklu', Deborah A. Levin, Sergey F. Gimelshein, and Joanna M. Austin

Citation: 1786, 050004 (2016); doi: 10.1063/1.4967554

View online: http://dx.doi.org/10.1063/1.4967554

View Table of Contents: http://aip.scitation.org/toc/apc/1786/1

Published by the American Institute of Physics 


\title{
Modeling of Near-Continuum Laminar Boundary Layer Shocks Using DSMC
}

\author{
Ozgur Tumuklu ${ }^{1, \text { a) }}$, Deborah A. Levin ${ }^{1}$, Sergey F. Gimelshein ${ }^{2}$ and Joanna M. \\ Austin $^{3}$ \\ ${ }^{1}$ Department of Aerospace Engineering, University of Illinois at Urbana-Champaign \\ ${ }^{2}$ Department of Astronautical Engineering, University of Southern California, Los Angeles \\ ${ }^{3}$ Department of Aerospace Engineering, California Institute of Technology \\ a)Corresponding author: tumuklu2@illinois.edu
}

\begin{abstract}
The hypersonic flow of nitrogen gas over a double wedge was simulated by the DSMC method using two-dimensional and three-dimensional geometries. The numerical results were compared with experiments conducted in the HET facility for a high-enthalpy pure nitrogen flow corresponding to a free stream Mach number of approximately seven. Since the conditions for the double wedge case are near-continuum and surface heat flux and size of the separation are sensitive to DSMC numerical parameters, special attention was paid to the convergence of these parameters for both geometries. At the beginning of the simulation, the separation zone was predicted to be small and the heat flux values for the 2-D model were comparable to the experimental data. However, for increasing time, it was observed that the heat flux values and shock profile strongly deviated from the experiment. Investigation of a three-dimensional model showed that the flow is truly three-dimensional and the side edge pressure relief provides good agreement between simulations and the experiment.
\end{abstract}

Keywords: Shock-wave boundary layer interactions, hypersonic separated flows, DSMC

\section{INTRODUCTION}

To accurately predict shock wave boundary layer interactions (SWBLI) for different flow and geometry configurations, many experimental and CFD numerical studies have been conducted in the past by various researchers due to their significant impact on aerothermodynamic quantities such as heat transfer, skin friction and surface pressure coefficient. Edney [1] classified six different shock interactions according to experimental observations on oblique and bow shock waves and also observed that extraneous shocks that impinge on the surface can cause extreme heating and pressure loads around the interaction points. Yet another type of shock interaction occurs in a double-wedge configuration studied by Olejniczak when the angle of the second wedge increases, thus causing pressure oscillations near the boundary of the aftbody [2, 3]. Further studies were designed by Holden and Wadhams [4] to obtain detailed measurements for code validation for laminar flows with shock wave-boundary layer and shock-shock interactions over hollow cylinder/flare and bi-conic configurations. In addition, Rudy et al. [5] showed that three-dimensional effects become important especially in a sharp wedge of which span is comparable with the separation zone size for a Mach number of approximately 14 and a Reynolds number of 240,000. Their three-dimensional CFD simulations were found to be in good agreement with the experimental measurements of the size of the separation zone. Furthermore, the separation zone predicted by the three-dimensional calculations was found to be smaller compared to that of a two-dimensional geometry and had a significant effect on the flow stability.

Experiments on a double-wedge configuration were conducted by Swantek and Austin [6] and the impact of thermochemical effects on shock wave-boundary layer interaction by changing the chemical composition from nitrogen to air and the stagnation enthalpy of flow were investigated. Experiments were carried out in the Hypervelocity Expansion Tube (HET), $9.14 \mathrm{~m}$ long expansion tube facility, consisting of a driver, driven, and accelerator section, all with an internal diameter of $150 \mathrm{~mm}$. The facility is capable of operating at Mach numbers of 3.5-7.5 and achieving stagnation enthalpies of 2-8.8 MJ/kg. To observe density gradients and track the transient shock profile in the flow, a

30th International Symposium on Rarefied Gas Dynamics

AIP Conf. Proc. 1786, 050004-1-050004-8; doi: 10.1063/1.4967554

Published by AIP Publishing. 978-0-7354-1448-8/\$30.00 
Z-type schlieren visualization system and image tracking code are used. In addition, the heat transfer is measured via coaxial nineteen fast response thermocouples mounted at the surface of a double wedge.

These experiments have motivated a number of recent numerical studies using continuum CFD [7,8] and Direct Simulation Monte Carlo (DSMC) [9] approaches and are part of the NATO RTO-AVT 205 program in the Assessment of Predictive Capabilities for Aerothermodynamics Heating of Hypersonic Systems. In this study, the high-enthalpy nitrogen case is considered and the flow conditions are presented in Table 1. Two-dimensional numerical calculations showed a different time dependence for the flow than was observed in the experiments for both high enthalpy and low enthalpy cases [6]. At the beginning of the simulation, the separation zone was predicted to be small and the heat flux values were comparable with the experimental data. However, for increasing time, it was observed that the triple point moved forward, the separation zone increased, and finally, at times longer than the duration of the experiments, the heat flux on the second wedge strongly deviated from the experiments of Austin.

To resolve this key discrepancy, we performed three-dimensional calculations using the DSMC method for the high enthalpy case. We choose the DSMC approach because when its numerical parameters are selected appropriately, it provides a statistical solution of the Boltzmann equation, which provides the most general and accurate description of dilute flows. Importantly, the Boltzmann equation is applicable in strong shock regions, where the continuum assumption breaks down. Hence, the DSMC method, although numerically expensive, provides a simulation capability valid throughout the flow, and for any flow regime from free molecular to continuum.

\section{NUMERICAL PARAMETERS}

In this work, numerical analyses were performed using the direct simulation Monte Carlo (DSMC) [10] method, a stochastic approach to solving the Boltzmann equation to model SWBLIs. The Statistical Modeling In Low-density Environment (SMILE) [11] computational solver was used in all 2D and 3D simulations presented below. This method has also been used to model SWBLIs, but, to a lesser extent than CFD due to its higher computational expense especially in the near-continuum regime. To obtain solutions that are independent of the selected numerical parameters such as time step, cell size, and the total number of particles, it is required that the time step be less than the mean time between collisions and the residence time of the particles in collisions cells. Furthermore, the collision cell size should be less than the mean free path and there are at least 3-4 particles per cell for those non-reacting cases. For the 2D case, the cell size is sufficiently small such that the local mean free path to collision cell size ratio is larger than unity throughout the domain except for a small region near the second wedge. A finer calculation was performed to ensure this ratio is larger than 1.2 in this and the separation region. It was shown that the velocity slip profiles using either case are the same, therefore, the former grid is sufficiently resolved [12]. On the other hand, for the 3D case, this ratio does not meet the strict DSMC convergence criterion. This as well as the lack of resolution at the wedge's edge means that the 3-D results that will be presented should be considered qualitative in nature. Therefore, a case in which the freestream number density is decreased by a factor of eight in comparison to the experiment is also presented and fully resolved in terms of any numerical parameters. The flow conditions and numerical parameters for this case are given in Tables 1 and 2 and are denoted "density decreased case".

With respect to the two remaining DSMC numerical parameters, the number of particles and time step, there are important differences between the 2D and 3D cases. Since the number of collision cells required to obtain a resolution on the order of a mean free path is high, the corresponding number of simulated particles becomes prohibitively large especially in 3D cases. The 2D simulation (experimental case) was performed using more than 1.1 billion simulated particles, the number of particles per collision cell in the shock region was found to be around four. This number is sufficient since the majorant collision frequency algorithm is used in the selection of collision pairs in this work [13]. For the three-dimensional cases, the number of simulated particles used was about 20 billion and the number of particles per collision cell in the shock region was about five. Therefore, a sufficient number of simulated particles was used in the 3D DSMC computations as well. Since the number of particles and collision cells and cells for these calculation are quite large, parallel implementation on approximately 10,000 CPUs were used.

Finally, in both the 2D and 3D simulations, a time step of $1.0 \times 10^{-9}$ and $4.0 \times 10^{-9} \mathrm{~s}$ were chosen such that the ratio of the mean collision time to the time step is greater than unity for the experimental and the density decreased case, respectively. As will be discussed in the next section, since the $2 \mathrm{D}$ results varied in time, a total of 800,000 and 200,000 time steps were computed for the experimental and density decreased case, respectively. In contrast, the 3D flowfields were found not to change significantly after 140,000 time steps $(140 \mu \mathrm{s})$, hence, a maximum of 200,000 and 100,000 time steps was computed the experimental and density decreased case, respectively. Even though the DSMC method is inherently time accurate, as compared to past continuum CFD approaches applied to the problem of 
TABLE 1. Free Stream Conditions

\begin{tabular}{lcc}
\hline \multicolumn{1}{c}{ Freestream Parameters: } & Experimental Case & Density Decreased Case \\
\hline Mach number & 7.14 & 7.14 \\
Translational temperature, $\mathrm{K}$ & 710 & 710 \\
Static pressure, $\mathrm{kPa}$ & 0.78 & 0.098 \\
Velocity, $\mathrm{m} / \mathrm{s}$ & 3812 & 3812 \\
Density, $\mathrm{kg} / \mathrm{m}^{3}$ & 0.0037 & 0.000465 \\
Number density, molec $/ \mathrm{m}^{3}$ & $7.96 \times 10^{22}$ & $1.0 \times 10^{22}$ \\
Stagnation enthalpy, MJ $/ \mathrm{kg}$ & 8.0 & 8.0 \\
Unit Reynolds number, Re/m & $4.15 \times 10^{5}$ & $5.22 \times 10^{4}$ \\
Mean free path, m & $2.05 \times 10^{-5}$ & $1.64 \times 10^{-4}$ \\
Knudsen number & $4.08 \times 10^{-4}$ & $3.24 \times 10^{-3}$ \\
\hline
\end{tabular}

TABLE 2. DSMC Numerical Parameters for the simulations

\begin{tabular}{ccc|ccc}
\hline & \multicolumn{2}{c}{ Experimental Case } & \multicolumn{3}{c}{ Density Decreased Case } \\
\hline Numerical Parameters: & $2-\mathrm{D}$ & 3-D & 2-D & $\begin{array}{c}\text { 3-D } \\
\text { Fully Resolved }\end{array}$ & $\begin{array}{c}\text { 3-D } \\
\text { Partially Resolved }\end{array}$ \\
\hline Total number of time-steps & 800,000 & 200,000 & 200,000 & 100,000 & 50,000 \\
Time step, s & $1.0 \times 10^{-9}$ & $1.0 \times 10^{-9}$ & $4.0 \times 10^{-9}$ & $4.0 \times 10^{-9}$ & $4.0 \times 10^{-9}$ \\
Number of simulated particles & $1.16 \times 10^{9}$ & $18.4 \times 10^{9}$ & $135 \times 10^{6}$ & $23.9 \times 10^{9}$ & $5.97 \times 10^{9}$ \\
Total number of collision cells & $3.05 \times 10^{8}$ & $3.92 \times 10^{9}$ & $25 \times 10^{6}$ & $5.92 \times 10^{9}$ & $1.04 \times 10^{9}$ \\
Number of processors (CPUs) & 960 & 6,400 & 256 & 10,240 & 3,200 \\
Total computation hours (CPU hours) & 46,080 & 853,330 & 2,048 & $1,260,000$ & 80,000 \\
\hline
\end{tabular}

interest $[7,8]$, the initial flow transients that occurred in the experiments were not modeled here. For the experimental case, the macroparameter sampling was started at 30,000 time step, and conducted in 40,000 increments between 40,000 and 200,000 steps and finally 100,000 increments after 200,000 step in order to observe the flow transients in the DSMC solution. Similarly, the corresponding time steps are selected for the latter case. It should be noted that the experiment was conducted with a test time of 242 micro-s.

\section{RESULTS AND DISCUSSION}

\section{Experimental Case}

The principal shock-shock interaction features obtained in the DSMC simulations are seen in the Mach number contours shown in Fig. 1. The figure shows the oblique shock formed at the first wedge which interacts with the detached bow shock from the second wedge. These outer shocks are further modified by a strong separation zone and the associated separation and reattachment shocks resulting in a flow field with complex features such as the triple point, shock impingement, shear layers and boundary layer interactions. The computed shock structure can be seen to be in qualitative agreement with the experimentally obtained Schlieren image in terms of the location and structure of the oblique shock, bow shock and their interaction, i.e., the triple point, transmitted shock, shear layer, separation and reattachment shock. To examine the evolution of the flow, shock tracking measurements using Schlieren flow visualization of the shock profile were obtained. The experimental shock tracking measurements [6] show that the movement of the triple point towards the tip of the wedge practically stops after $100 \mu \mathrm{s}$ for the duration of the experiment, $240 \mu \mathrm{s}$. However, when the 2-D DSMC simulations were continued beyond $100 \mu \mathrm{s}$, the translational temperature field did not come to steady state even at $300 \mu$ s, as seen in Fig. 2. Qualitatively similar behavior was also observed in the non-reacting NS simulations by Knight et al [7], where HET measurements in air were examined. The sequence of contours and streamline plots presented in Fig. 2 shows that the size of the separation bubble keeps on increasing and moves toward the leading edge, causing considerable changes in the shock structure, in contrast to the experiment. The 2D DSMC simulations were run for a total of $800 \mu \mathrm{s}$ and, in contrast to the experiment, it was observed that the flow reaches steady state in terms of the shock position only after approximately $500 \mu \mathrm{s}$. The time dependence of the 


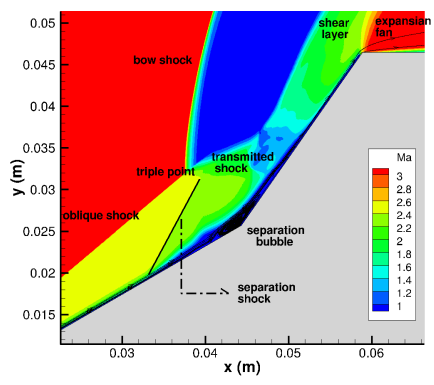

(a) Experimental case

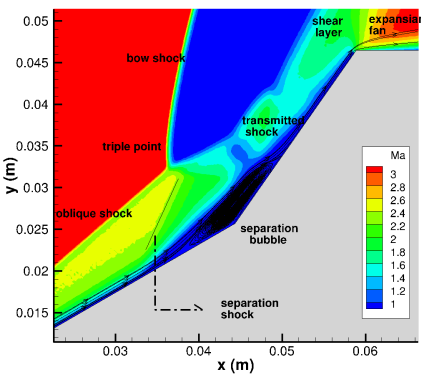

(b) Density decreased case

FIGURE 1. Shock structure and Mach numbers in the shock interaction region at $100 \mu \mathrm{s}$

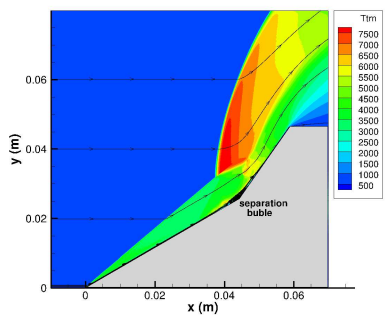

(a) $100 \mu \mathrm{s}$

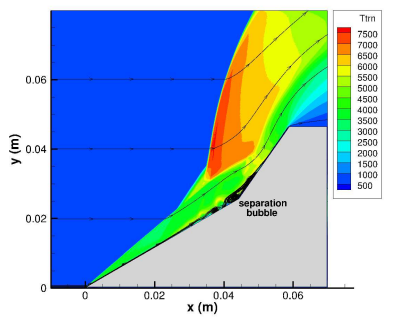

(b) $200 \mu \mathrm{s}$

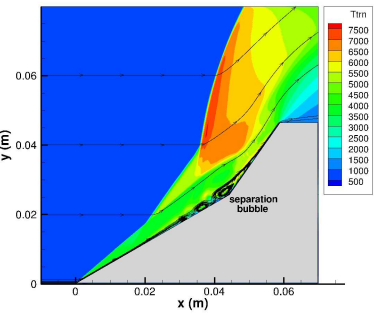

(c) $300 \mu \mathrm{s}$

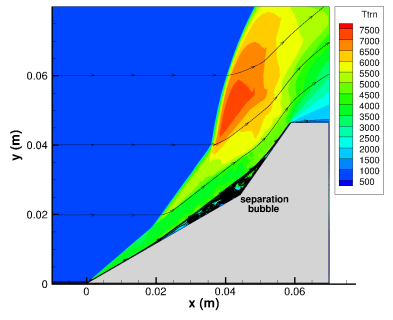

(d) $800 \mu \mathrm{s}$

FIGURE 2. Translational Temperature $(\mathrm{K})$ contours and streamlines for different computational times for the experimental 2-D case.

translational temperature is further illustrated in the line plot shown in Fig. 3(a), where the temperatures are extracted at a cross section $y=0.04 \mathrm{~m}$ which passes through the origin of the strong bow shock on the second wedge. As the figure shows, the temperature values change substantially in time due to significant changes in shock structure. It is not possible to compare translational temperatures directly with the experiment, however, as shown by Moss and Bird [12] comparison of DSMC results with experimentally obtained heat flux measurements is a good test of the simulation fidelity. Figure 3(b) shows comparison of the heat transfer rates obtained from the experiment [6] and our $2-\mathrm{D}$ DSMC simulations for different times. It can be seen that at $100 \mu \mathrm{s}$, the DSMC result is in reasonable agreement with the experiment, especially in the region close to the tip of the first wedge and at the second wedge surface, while the heat transfer values on the second wedge differ significantly in time. Indeed, at the beginning of the simulation, the computed heat flux spatial distribution is similar to the experiment, although the values are slightly over predicted. However, the differences between simulation and experiment grow larger in magnitude as time progresses, and by $800 \mu$ s shows a spatial pattern that is completely different from the experiment.

The lack of agreement between the time evolution of the simulations and the experiment suggests that the flow may not be two-dimensional in the $Z=0$ symmetry plane, although, the previous empirical results of Ball [14] of flow over a symmetric wedge/flap configuration under similar free stream conditions show that the span to boundary layer thickness at the hinge should have been sufficient to prevent edge effects from reaching the centerline. Hence, the goals of the 3-D simulations are to establish whether the flow in the $Z=0$ symmetry plane is affected by threedimensionality, and to what extent the inclusion of the flow in the spanwise $(Z)$ direction reduces the shock-shock interaction (pressure relief effect). A three dimensional view of the computational domain and the double wedge with the simulated translational temperature contours superimposed is shown in Fig. 4(a). As can clearly be seen in Fig. 4(b) the flow is three-dimensional, since the translational temperature contours are strongly non-uniform in the $z$-direction. Similarly, the top view of the chemiluminescence image shown in Fig. 4(c) indicates that the high temperature region differs from the wide centerline region to the edge. In Fig. 4(d), the streamlines at the wedge surface are shown. Below 


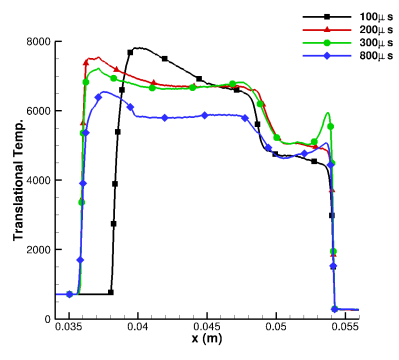

(a) Temperature $(\mathrm{K})$ profile along $y=0.04 \mathrm{~m}, 2-\mathrm{D}$

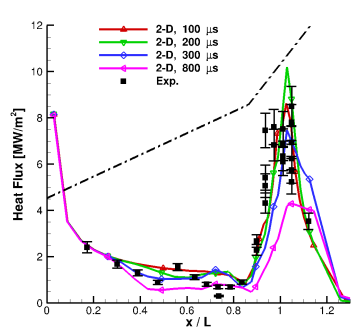

(b) Heat transfer rate, 2-D
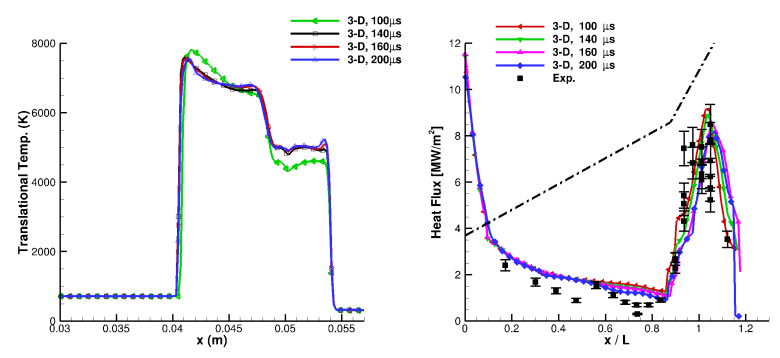

(c) Temperature $(\mathrm{K})$ profile along(d) Heat transfer rate, 2-D at the cen$y=0.04 \mathrm{~m}, 3-\mathrm{D}$, at the center ter

FIGURE 3. Variation of temperature and heat transfer values in time.

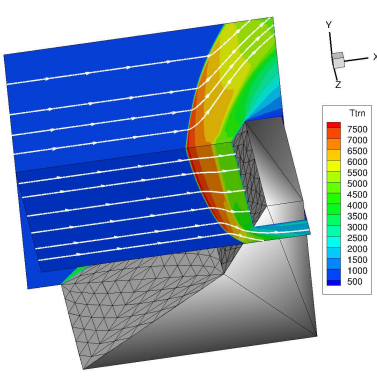

(a) 3D view

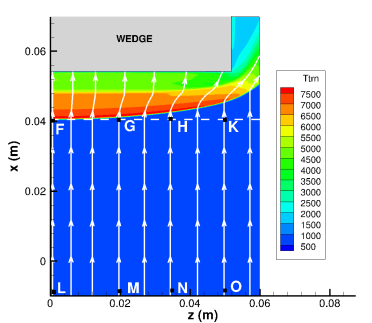

(b) Top view at $y=0.04 \mathrm{~m}$ constant(c) Chemiluminescence image (top plane

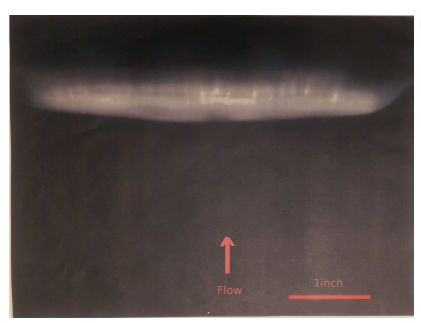
view)

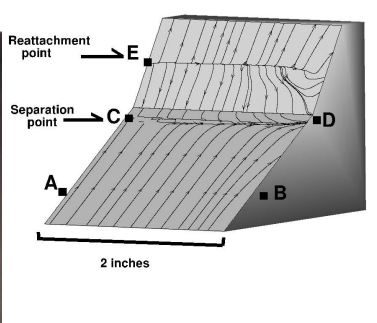

(d) Streamlines on the surface

FIGURE 4. The 3D effects at $140 \mu$ s simulated using the 3-D (baseline case) wedge geometry. The backplane is the $Z-0$ symmetry plane.

the $\mathrm{AB}$ line, the velocity field has almost no $Z$-component. However, the flow crossing the $\mathrm{C}$ and $\mathrm{D}$ line shows strong three-dimensionality, with the streamlines directed towards the outer edge of the wedge in the $Z$ direction. These results are consistent with the CFD simulations reported earlier by Rudy et al. [5].

The effect of the three-dimensionality of the flow on its time dependence along the center region is examined next. Figure 3(c) presents the translational temperature profiles as a function of time at a $Y$ plane passing through the separation region. As shown, the temperature profiles only slightly change in time, especially after $140 \mu \mathrm{s}$. In fact, the extent of the shock region is almost the same, and the temperature variation as a function of time is insignificant in the shock region, in direct contrast to the two dimensional case for 100 and $200 \mu$ s presented in Fig. 3(a), where both the extent of the shock and temperature values were observed to change dramatically in time. Figure 3(d) shows the heat transfer rate obtained from experiment (including error bars with about 10\% deviation from its mean) and the 3-D calculation. The heat flux decreases along the first wedge from the free stream to the hinge point, as predicted by the laminar boundary-layer theory [15]. The calculated heat flux rates are found to be in reasonable good agreement with the measurements on the first wedge, however, the solution does not show any indication of the separation along the first wedge. This is likely due to the poorer cell and panel resolutions especially at the location, $x / L=0.8$. Nonetheless, much better agreement with the experiment is achieved at the aft part of the wedge compared to the 2-D result.

SWBLI is susceptible to three dimensional instabilities and is sensitive to the dimensionality of the flow. The comparison of the difference in the predicted shock structure along the wedge centerline for the 2 and 3-D geometries shows the boundary layer for both geometries becomes wider at the point where the separation shock is formed. Additionally, this point is closer to the tip of the wedge in the 2-D case which results in a larger separation region compared to the 3-D case. Also, for the 2-D case, the inside area surrounded by the bow shock and transmitted shock and the shear layer is larger in 2-D compared to the 3-D case. 


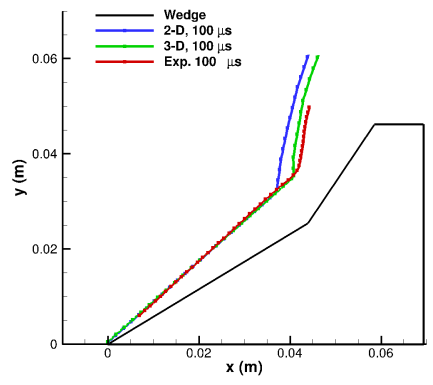

(a) $100 \mu \mathrm{s}$

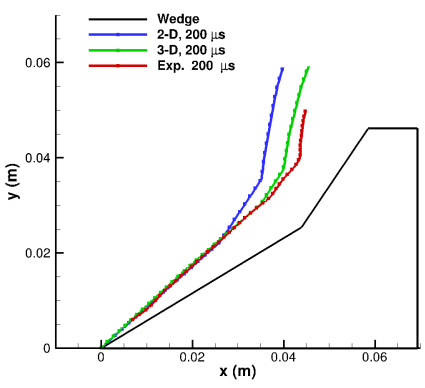

(b) $200 \mu \mathrm{s}$

FIGURE 5. Comparison of the position of the shock wave between simulations and experiment for the 2 and 3-D geometries.

To understand the difference in the predicted temporal variation of the two and three-dimensional flows, we compare the translational temperature profiles, streamlines and shock structure profiles of these two models. We found that the degree of thermal nonequilibrium for rotation and vibration did not reveal any significant difference between the two or three-dimensional model. Early in the time evolution of the flow, $40 \mu \mathrm{s}$, the translational temperature spatial distribution was found to be the same for both the two and three-dimensional models. Generally at this time, there is no significant difference between the two cases since the size of the separation zone is not large and surface streamlines show little variation in the spanwise direction. The length of the high temperature region in the 2-D case was found to be slightly larger than in 3-D due to the fact that the triple point is slightly forward compared to the 3-D case. As time progresses, the separation zone moves towards the tip of the wedge and becomes larger in the 2-D case. The difference between the 2-D and 3-D flow structures becomes significant in time. As can be seen in Fig. 5, in the 2-D case, the triple point continues to move and the difference becomes larger. Indeed, the 3-D calculations are in good agreement with the experimental result. A small deviation of about $1 \mathrm{~mm}$ in the experimental tracking data may be attributed to the uncertainty in the image tracking and the shock blurring that occurs due to the finite shutter time and pixel resolution.

\section{Density Decreased Case}

The previously presented three-dimensional results are not fully resolved in terms of the DSMC cell criterion in the streamwise as well as spanwise directions. That is, the ratio of local mean free path to the cell size is smaller than unity especially in the separation region and at the edge of the wedge. Therefore, the freestream density value is decreased by a factor of eight to solve the problem without assuming the gradient in the z-direction is negligible and to ensure that this ratio is larger than unity. The shock structure shown in Fig. 1(b) is found to be somewhat similar to that in the experimental case shown in Fig 1(a) in terms of the shock interactions and the existence of the distinctive separation region. By using the same geometry and same flow conditions (i.e. Mach number, stagnation enthalpy), this case at least provides the opportunity to show the three-dimensional effects on the double wedge configuration in a fully resolved manner. Comparison of the shock structure with the experimental case reveals that the boundary layer is thicker based on the fact that Reynolds number is lower than the experiment, resulting in the larger separation region. The position of the transmitted shock for both cases is found to be similar where the peak of heat transfer rate occurs. Consistent with the decrease in the freestream density, the surface heating values for the density decreased case is decreased by about a factor of four as compared with the experiment.

Similar to the experimental case, it was observed that the size of the separation bubble keeps on increasing and moves toward the leading edge, causing significant changes in the shock structure for the 2-D case. As shown in Fig. 6(a), the temperature values are extracted along the $y=0.04$ constant plane and found to change substantially in time similar to those in Fig. 3(a). The effect of the change in the temperatures can also be seen in the heat flux values shown in Fig. 6(b). The effect of the separation is to lower the heat transfer rate and is more distinctive in comparison to the experimental case. This is due to the fact that the heat flux values are lower which makes it more sensitive to the small changes. In contrast to the 2-D case, the temperature profiles shown in Fig. 6(c) slightly change in time and are 


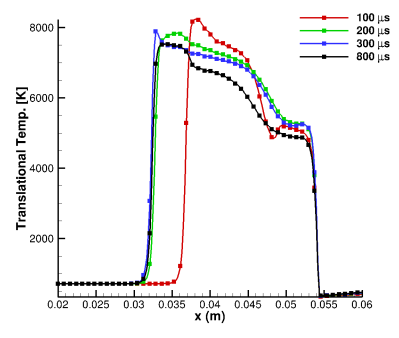

(a) Temperature $(\mathrm{K})$ profile along $y=0.04 \mathrm{~m}, 2-\mathrm{D}$

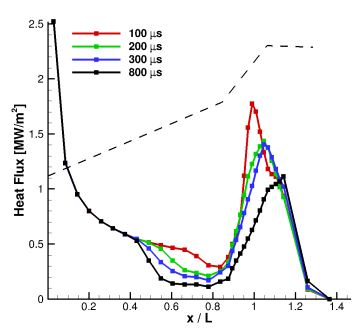

(b) Heat transfer rate, 2-D
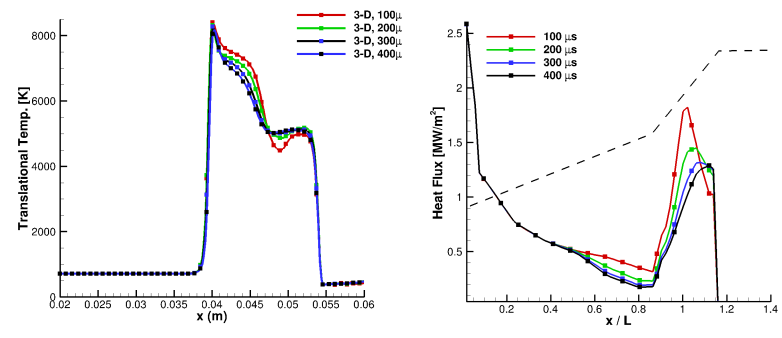

(c) Temperature (K) profile along(d) Heat transfer rate, 2-D at the cen$y=0.04 \mathrm{~m}, 3-\mathrm{D}$, at the center

ter

FIGURE 6. Variation of the temperature and heat transfer values in time for the density decreased case.

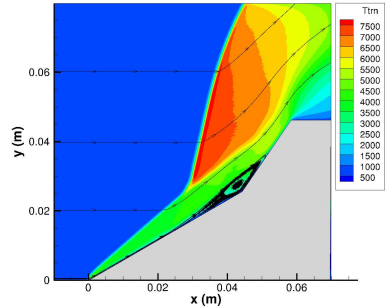

(a) 2-dimensional case
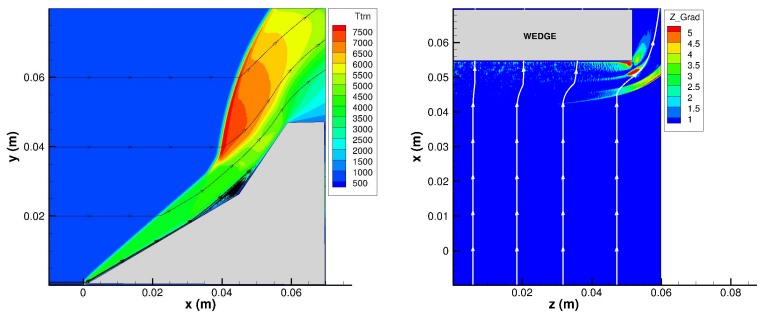

(b) 3-dimensional case at the center(c) Gradient values in the z-direction plane

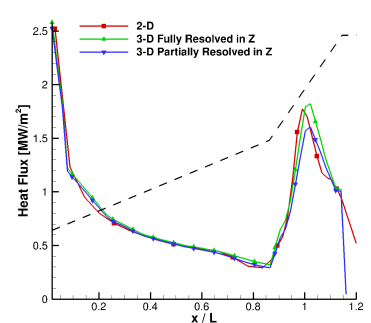

(d) Heat transfer of degraded case

FIGURE 7. Comparison of translational temperature $(\mathrm{K})$ contours and heat transfer rates as a function of dimensionality at $100 \mu \mathrm{s}$.

found to be stable in terms of the shock location for the 3-D case. However, heat flux values continue to change in time as shown in Fig. 6(d). As opposed to the experimental case, there is a significant decrease of the maximum between 100 and 200 micro-s which is more likely due to the existence of thicker boundary layer and larger separation region in the density decreased case. Consistent with the previous observation, the separation starts closer to the leading edge in the 2-D than the 3-D case due to the side edge pressure relief effects. The effect of the three-dimensionality is more obvious in the translational temperature profiles at $400 \mu$ s shown in Figs. 7(a) and 7(b). In fact, the high-temperature region after the strong detached shock is larger in the 2-D case.

The lower density case also provides opportunity to test and validate the assumption of the use of larger collision cell in the spanwise direction based on the low gradient values shown in Fig. 7(c). The cell division in the z-direction was restricted in the experimental case and this provides the reduction of computational requirements by a factor of 40 whereas for the density decreased case, this number is about 4 as presented in Table 2 . The translational temperature values both at the center and the edge are found to be essentially the same. However, as can be seen in Fig. 7 (d) at most $10 \%$ differences are observed at the peak of the heat values which is in the limit of experimental uncertainty. Therefore, larger cells can be used in the spanwise direction. It should be noted that at $100 \mu$ s the heat flux values obtained for all lower density cases are close to each other, showing that the numerical parameters are set accurately.

\section{CONCLUSIONS}

The hypersonic flow of nitrogen gas over a double wedge was simulated by the DSMC method using two dimensional and three dimensional geometries. The numerical results were compared with experiments conducted in the HET facility for a high-enthalpy pure nitrogen flow corresponding to a free stream Mach number of approximately seven. When the flow was assumed to be two-dimensional, the shock structure and flowfield properties were shown to continue to change in time, in contrast to the experiment, due to the growing separation zone near the hinge. Al- 
though the 2-D solution at $100 \mu$ s qualitative agrees with the experiment in terms of heat transfer rates and Schlieren visualization, the deviation of from the experiment grew as the simulations were continued in time.

Three-dimensional simulations were performed to investigate the three dimensionality effect on the flowfield and on the stability of the shock structure locations. It was observed that at the beginning of the simulation, when the separation zone is not distinct, three-dimensional effects are not significant. This causes the 2-D and 3-D results to be similar early in time. However, the differences grow in time due to changes in the separation region. Also, it was seen that the flowfield and surface properties differ significantly between the edge and the center plane, although the flow in the spanwise direction is not modeled accurately due to the poorer grid resolution in the $\mathrm{Z}$ direction. The simulated 3-D heat fluxes, shock structure, and triple point movement were found to be in excellent agreement with the experimental heat flux values, especially in the aft part of the wedge, and the tracking measurements.

The validity of the assumptions made in the experimental case was tested by the lower density case. It was observed that the shock structure and separation region are found to be similar for both cases. However, the time trend observed in the peak of heat flux can be attributed to the differences in the boundary layer associated with different Reynolds number between the two cases. The three-dimensional effects are significant enough to change the flow characteristics.

\section{ACKNOWLEDGMENTS}

The research is being performed at the University of Illinois Urbana-Champaign is supported by the Air Force Office of Scientific Research through AFOSR Grant No. FA9550-11-1-0129 with a subcontract award number 2010-0617101 to UIUC. This research is also a part of the Blue Waters sustained-petascale computing project, which is supported by the National Science Foundation (awards OCI-0725070 and ACI-1238993) and the state of Illinois. Blue Waters is a joint effort of the University of Illinois at Urbana-Champaign and its National Center for Supercomputing Applications.

\section{REFERENCES}

[1] B. E. Edney, AIAA Journal 6, 15-21 (1968).

[2] J. Olejniczak, M. J. Wright, and G. V. Candler, Journal of Fluid Mechanics 352, 1-25 (1997).

[3] J. Olejniczak, M. J. Wright, and G. V. Candler, "Numerical study of shock interactions on double-wedge geometries," AIAA paper 96-0041 (1996).

[4] M. S. Holden, T. P. Wadhams, G. V. Candler, and J. K. Harvey, "Measurements in regions of low density laminar shock wave/boundary layer interaction in hypervelocity flows and comparison with Navier-Stokes predictions," AIAA paper 2003-1131 (2003).

[5] D. H. Rudy, J. L. Thomas, A. Kumar, P. A. Gnoffo, and S. R. Chakravarthy, AIAA Journal 29, 1108-1113 (1991).

[6] A. Swantek and J. Austin, AIAA Journal 53, 311-320 (2014).

[7] M. Badr and D. Knight, "Shock wave laminar boundary layer interaction over a double wedge in a high mach number flow," AIAA Paper 2014-1136 (2014).

[8] J. R. Komives, I. Nompelis, and G. V. Candler, "Numerical investigation of unsteady heat transfer on a double wedge geometry in hypersonic flows," AIAA Paper 2014-2354 (2014).

[9] V. N. Patil, D. A. Levin, S. G. Gimelschein, and J. M. Austin, "Study of shock-shock interactions for the het facility double-wedge configuration using the dsmc approach," AIAA paper 2013-3202 (2013).

[10] G. A. Bird, in Molecular Gas Dynamics and the Direct Simulation of Gas Flows (Clarendon, Oxford, England, U.K., 1994).

[11] M. S. Ivanov, G. N. Markelov, and S. G. Gimelshein, "Statistical simulation of reactive rarefied flows numerical approach and applications," AIAA paper 1998-2669, AIAA/ASME Joint Thermophysics and Heat Transfer Conference, 7th, Albuquerque, NM, June 15-18 (1998).

[12] J. N. Moss and G. A. Bird, AIAA Journal 43, 2565-2573 (2005).

[13] M. S. Ivanov and S. V. Rogasinsky, Soviet Journal of Numerical Analysis and Mathematical Modeling 3, 453-465 (1988).

[14] K. Ball, AIAA Journal 9, 2080-2081 (1971).

[15] M. J. Hayne, D. J. Mee, S. L. Gai, and T. J. McIntyre, Journal of Thermophysics and Heat Transfer 21, 772-779 (2007). 